\title{
Joint Source and Channel Matching with Channel Estimation for Robust Image Transmission over Wireless Channel
}

\author{
Xiaowen Wang \\ Agere Systems \\ Murray Hill, NJ 07974 \\ xiaowenw@agere.com
}

\author{
Jie Song \\ Agere Systems \\ Homdel, NJ 07733 \\ jiesong@agere.com
}

\author{
K. J. Ray Liu \\ Uiversity of Maryland \\ College Park, MD 20742 \\ kjrliu@eng.umd.edu
}

\begin{abstract}
Joint source and channel coding (JSCC), multicarrier modulation (MCM) and diversity are some key techniques for the multimedia transmission over wireless channels. In this work, we first investigate the channel estimation problem for the MCM system with multiple transmitter antennas. A model based channel estimation approach is proposed to identify the multiple channels simultaneously. We then apply the model based channel estimation in a joint source and channel matching (JSCM) scheme for the robust progressive image transmission. It is shown that the channel estimation affects not only the image decoding at the receiver but also the rate allocation at the transmitter. It is important to jointly consider the channel estimation and JSCM scheme to improve the quality of image transmission.
\end{abstract}

\section{INTRODUCTION}

To support the broadband applications such as image and video in wireless communcaitons, one challenge is to combat the serious impairment of the wireless channel. Multicarrier modulation (MCM) is considered as an effective technique for wireless broadband communications [1] for its great resistance to the inter-symbol interference (ISI) caused by the multipath effect because of its relative longer symbol duration. The other serious impairment of the wireless channel is the fading effect. To combat fading, multiple antennas can be used at the receiver and/or at the transmitter, which are known as receiver diversity and/or transmitter diversity, respectively, to collect different copies of signal in order to enhance the received signal power. It has been proved in many works [9] that the channel capacity is proportional to the number of the transmitter or receiver antennas.

In this paper, we use the MCM system with diversity to provide a robust pipe for image transmission. In [11], it is proved that the subchannels in a MCM system with sufficient diversity can be viewed as a nonfading AWGN channels. Then we can apply various sophisticated joint source and channel coding techniques originally designed for AWGN channel for multimedia transmission. One such joint source channel matching (JSCM) is used in [11] to transmit image progressively through wireless channel. In the algorithm, the image is encoded progressively [12], [13], then different channel codes are selected to provide unequal error protection according to the importance of the encoded irnage. However, like most research on joint and source channel coding, the channel responses are assumed perfectly known to the receiver and the corresponding receiver performance is used for the selection of the channel codes. Such an assumption is not true in practice. The receiver has to use a channel estimation algorithm to get the channel responses. In this paper, we investigate the channel estimation problem for such a system and the effect of the channel estimation on image transmission using JSCM by applying the channel estimation results at both the transmitter and the receiver.

Various channel estimation schemes are proposed for various communication systems [3], [6], mostly for single antenna system. The challenge of the channel estimation for the system with transmitter diversity is that we have to estimate multiple sets of channel responses from the same set of observations as that in the single transmitter antenna case. Our solution is to exploit the correlation among channel responses by using some efficient channel model and projecting a larger set of channel responses onto a smaller set of model bases. Thus, small number of model coefficients need to be estimated instead of the large number of channel responses. The estimation noise is hence greatly reduced.

Based on the proposed channel estimation scheme, we apply it in the robust progressive image transmission using JSCM. The simulation shows that the channel estimation scheme has a great impact to the result of the JSCM algorithm. It is important to jointly consider channel estimation when design such joint source channel coding algorithm.

\section{MCM SYSTEMS WITH TRANSMITTER DIVERSITY}

In a MICM system with $m_{T}$ transmitter antennas and $m_{R}$ receiver antennas, one block of data symbols comes into the encoder and forms $m_{T}$ blocks of MCM symbols to be modulated simultaneously. From the $i$ th transmitter antenna to the $j$ th receiver antenna, the $n$th block 
of data generated by the space-time encoder consists of $K$ subsymbols $X_{i}[n, k], k=0, \cdots, K-1$. Then the modulation is implemented by $K$-point inverse discrete Fourier transform (IDFT). The modulated signal passes through a $\mathrm{P} / \mathrm{S}$ converter and a cyclic prefix of length $v$ is inserted before sending it to the channel. At the receiver, the cyclic prefix part is discarded. The demodulation then is performed by discrete Fourier transform (DFT). If the suchannel number is large enough and the cyclic prefix is long enough, then the subchannels can be viewed as independent of each other, i.e., the received signal $Y_{j}[n, k]$ of $k$ th subchannel in $n$th block at $j$ th receiver antenna is

$$
Y_{j}[n, k]=\sum_{i=1}^{m_{T}} H_{i j}[n, k] X_{i}[n, k]+\Upsilon_{j}[n, k],
$$

for $j=1, \cdots, m_{R}$, where $H_{i j}[n, k]$ is channel response from the $i$ th transmitter antenna to the $j$ th receiver. $\Upsilon_{j}[n, k]$ is the noise at the $j$ th receiver antenna. The noise is assumed to be white Gaussian noise with zero mean and variance of $\sigma^{2}$ and independent with different $n$ 's, $k$ 's and $i$ 's, $j$ 's.

The detection and decoding are done over $Y_{j}[n, k]$ 's. The general detection metrics is

$$
d=\sum_{n} \sum_{k} \sum_{j=0}^{m_{R}}\left\|\sum_{i=1}^{m_{T}} Y_{j}[n, k]-H_{i j}[n, k] X_{i}[n, k]\right\|^{2} .
$$

The detected signal is the transmit sequence that minimize this metrics. Different detection techniques are developed according to different space-time coding schemes. However, the channel responses $H_{i j}[n, k]$ 's are all necessary for coherent decoding which is used by most of space-time codes. Moreover, it is obvious that at each receiver antenna we need to estimate $m_{T}$ sets of channel responses $H_{i j}[n, k]$ 's for $i=1,, \cdots, m_{T}$, which make the channel estimation problem more complicated.

We assume that all the channels from different transmitter antennas to different receiver antennas have same global statistical property, i.e., same delay and fading property.

\section{Model Based Channel Estimation}

In this section, we begin to consider the channel estimation problem at $j$ th receiver antenna. The estimation scheme developed here can be applied at all receiver atennas to obtain all the channel estimations. From now on, we will omit the subscript $j . H_{i}[n, k]$ denotes the channel response from the $i$ th transmitter antenna to the receiver antenna.
We assume that $m_{T}$ training sequences $t_{i}[k]$ 's are sent simultaneously from all transmitter antennas. Then considering a window of $L$ received symbols $\mathbf{Y}[n, k]=$ $[Y[n, k] Y[n, k+1] \cdots Y[n, k+L-1]]^{T}$, write (1) in the matrix form we have

$$
\mathbf{Y}[n, k]=\mathbf{A}[k] \mathbf{H}[n, k]+\mathbf{\Upsilon}[n, k],
$$

where $\mathbf{A}[k]=\left[\mathbf{A}_{1}[k] \mathbf{A}_{2}[k] \cdots \mathbf{A}_{m_{T}}[k]\right]$ and $\mathbf{H}[n, k]=$ $\left[\mathbf{H}_{1}^{T}[n, k] \mathbf{H}_{2}^{T}[n, k] \cdots \mathbf{H}_{m_{T}}^{T}[n, k]\right]^{T}$, with

$$
\mathbf{A}_{i}[k]=\operatorname{diag}\left(t_{i}[n, k], t_{i}[n, k+1], \cdots, t_{i}[n, k+L-1]\right)
$$

and

$\mathbf{H}_{i}[n, k]=\left[H_{i}[n, k] H_{i}[n, k+1] \cdots H_{i}[n, k+L-1]\right]^{T}$,

for $i=1,2, \cdots, m_{T}$.

If we only have one transmitter antenna, then $\mathbf{A}[k]$ and $\mathbf{H}[n, k]$ degenerate to $\mathbf{A}_{1}[k]$ and $\mathbf{H}_{1}[n, k]$. Because $\mathbf{A}_{1}[k]$ is a square matrix, we can get a channel estimate directly from (3) as $\tilde{\mathbf{H}}_{j}[n, k]=\mathbf{A}_{1}^{-1}[k] \mathbf{Y}_{j}[n, k]$. However, now we need to estimate $m_{T}$ sets of channel parameters related to different transmitter antennas. The number of parameters required to estimate becomes $m_{T} L$ which is $m_{T}$ times of that in the single transmitter antenna case, but with same amount of $L$ observations. By no means we can directly estimate $\mathbf{H}_{i}[n, k]$ from (3).

Our solution is to use an efficient model to exploit the correlation of the channel. We try to project a large number of channel parameters to a small number of basis functions $\mathrm{p}(\mathrm{f})$, and expressed by the coefficients of the model basis, $b_{i}^{j}[n, k]$ 's, $H_{i}[n, k]=\sum_{j=0}^{l-1} b_{i}^{j}[n, k] p(j \Delta f)$. If the channel responses $H_{i}[n, k]$ 's are correlated, we can find a model basis $p(f)$ that we can express $\mathbf{H}_{i, j}[n, k]$ as

$$
\mathbf{H}_{i}[n, k] \simeq \mathbf{Q b}_{i}[n, k],
$$

where $\mathbf{Q}$ is a $L \times l$ matrix consisting of the model basis and $\mathbf{b}_{i}[n, k]=\left[b_{i}^{0}[n, k] b_{i}^{1}[n, k] \cdots b_{i}^{l-1}[n, k]\right]^{T}$.

The problem now is to find the model basis that can express the channel responses with less model coefficients and small model error. In ideal case, we know the correlation matrix of the channel responses defined as $\mathbf{R}_{H}=\mathrm{E}\left[\mathbf{H}_{i}[n, k] \mathbf{H}_{i}^{H}[n, k]\right]$. The model basis $\mathbf{Q}$ is the matrix consists of the eigen-vectors of the correlation matrix that corresponding to non-zero eigen-value of $\mathbf{R}_{H}$. However, the correlation of the channel responses is usually not available. We need to look for some model basis that can have a fairly small model error for most or some types of the real channels. We study two such model basis in this paper, one is Fourier basis, $(\mathbf{Q})_{m n}=\frac{1}{L} e^{-j \frac{2 m n \pi}{L}}$, for $m=0, \cdots, L-1$ and 
$n=0, \cdots, l-1$. Another is polynomial model basis, $(\mathbf{Q})_{m n}=(m-L / 2)^{n}$ for $m=0, \cdots, L-1$ and $n=0, \cdots, l-1$.

Suppose we find some efficient and accurate model and express the channel as (4). If the model is also efficient enough that $m_{T} l<L$, substituting it in (3), then we can get the LS estimation of model coefficients and construct the channel estimation as

$$
\hat{\mathbf{H}}[n, k]=\mathcal{Q R} \mathcal{R}^{-1}[k] \mathcal{Q}^{H} \mathcal{P}[n, k],
$$

where $\mathcal{Q}=\left[\begin{array}{cccc}\mathbf{Q} & \mathbf{0} & \cdots & \mathbf{0} \\ \mathbf{0} & \mathbf{Q} & \cdots & \mathbf{0} \\ \vdots & & \ddots & \vdots \\ \mathbf{0} & \mathbf{0} & \cdots & \mathbf{Q}\end{array}\right]$,

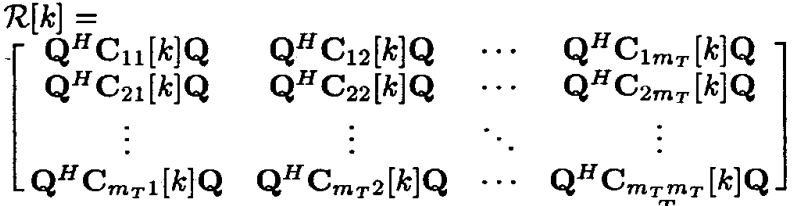
$\mathcal{P}[n, k]=\left[\begin{array}{llll}\mathbf{P}_{1}[n, k] & \mathbf{P}_{2}[n, k] & \cdots & \mathbf{P}_{m_{T}}[n, k]\end{array}\right]^{T}$, with $\mathbf{C}_{i j}[n, k]=\mathbf{A}_{i}[k] \mathbf{A}_{j}^{H}[k]$ is the correlation of the transmitted signal and $\mathbf{P}_{i}[n, k]=\mathbf{A}_{i}^{H}[k] \mathbf{Y}[n, k]$ is the crosscorrelation between the transmitted and received signals.

It can be seen from (5) that the channel can only be identified when $\mathcal{R}^{-1}[k]$ exists. This is called identification condition. The necessary condition is that $l \leq \frac{L}{m_{T}}$, which means that the channel model has to be efficient enough. On the other hand, the efficient enough model has to be accurate enough. For a fixed model, how efficient we can express the channel then depends on the channel characteristics and the model error allowed. It is usually required the model error to be much less than the noise. If the channel changes too fast, there is no efficient enough model to approximate it within the allowed model error and the channel is not identifiable. In this case, the training sequences from different transmitter antennas have to be sent at different time, which increases the overhead of the system. Moreover, the structure of the model alone can not guarantee the identification condition. Proper training sequences need to be carefully chosen carefully.

\section{Robust Progressive Image Transmission}

The subchannels in MCM systems with enough transmitter diversity can be viewed as AWGN channels [11]. Therefore many sophisticated techniques originally designed for AWGN channels can be applied to MCM systems with transmitter diversity. Here, we look at a joint source channel matching (JSCM) scheme for robust pro- gressive image transmission. The image coding algorithm used is SPIHT (Set Partitioning In Hierarchical Tree) [13' which is a refined version of embedded zerotree algorithm [12]. The output of the SPIHT encoder is progressive such that whenever the transmission is stopped, the received data can be used to reconstruct an image with corresponding quality.

The SPIHT bit-stream is packetized into sourcepackets of size $b_{n}$ bits for packet $n$. The source packets are channel-coded by the assigned channels codes and transmitted over the noisy channel. RS channel code is used to protect the source packets because of its burst error correction capability. Each MCM block. $n$ is a RS codeword $R S\left(K, k_{n}\right)$ over $G F\left(2^{m}\right)$, where $k_{n}=\frac{b_{n}}{m}$ is the RS information symbols in the MCM block. If the first $N$ scurce-packets are correctly received, the image can be reconstructed to a rate $R=\frac{\sum_{n=1}^{N}\left(k_{n} m-16\right)}{D_{H} D_{W}} \mathrm{bpp}$, , where $D_{H} \times D_{W}$ is the dimension of the irnage. According to the progressive source coding, the important packet has to be transmitted correctly in order: to decode the subsequent packets. This means that we need add more error protection to ensure that the correct transmission of the important packet. On the other hand, in order to get a higher resolution image, we need need to transmit source coding bits.

If we know some performance measure of the image, $D(R)$, such as the Peak-Signal-to-Noise-Ratio (PSNR), then the JSCM algorithm is to look for a source packetization scheme $\mathcal{A}$, which specifies the sequence of sourcepacket size as $\left\{k_{1} m, k_{2} m, \cdots, k_{L} m\right\}$ to optimize the performance $D(R)$ of the reconstructed image. In order to do this, we want this performance measure can be calculated easily. One practical choice is to use the expected number of source bits correctly received as suggested in [10] where dynamic programming is used to solve the optimization problem. Even though the use of the number of received bits is not as precise as using PSNR or ratedistortion function, the performance of using expected received bits is almost as good as using PSNR or ratedistortion function. The major difference between the JSCM we proposed here and the others is that, in our scheme, the channel codewords are of equal length, and we look for the length of source packet in each codeword, while the source-packets are of equal length in the algorithms used in most of current literatures. Dynamic programming can be used to find the optimal source packet size $b_{n}, n=1,2, \cdots, N$ that maximize the correctly received source bits subject to the total delay constraint $N$.

The other important information need to calculate the expected received bits is the error rate of RS code. In 
[11], the channel information is assumed perfectly known at the receiver, then the transmitter used a formula of RS error rate for AWGN channel to get this information. When channel estimation is used at the receiver, such a calculation becomes incompitable with the receiver and in the simulation we will show that such a discrepancy may cause server performance loss. The effect of the channel estimation has to be considered in the JSCM algorithm.

\section{Simulation Results}

In this section, we show some simulation results for the model based channel estimation method. The bandwidth of the system is $800 \mathrm{kHz}$. The number of the subchannels is 128 . The length of the cyclic prefix is 32 . The 4 subchannels of each end of a block are used as guarding band. The system uses 2 transmitter antennas and 2 receiver antennas. Training blocks are sent periodically from both transmitter antennas. After that, the channel estimate is used for decoding the data blocks arriving subsequently. We show the results of different training densities. The PSK constellation is used for all the subchannels. The training sequence is chosen as $t_{1}[k]=a$ and $t_{2}[k]=-a$, where $a$ is any constellation point. We did simulation the space time block code in [8] using 8PSK. The decoding scheme in [7] is adopted for decoding. Rayleigh fading channel with Doppler shift of $40 \mathrm{~Hz}$ is used in the simulation. TU delay profile [14] is used in the simulation.

Fig. 1 shows the mean square estimation error for the TU delay profile in which we also show the results of the 2-ray delay profile with the two paths separated by the $5 \mu$ which is the maximal delay of the TU profile. As shown in these figures, the polynomial model has lower estimation error as the SNR goes higher for TU while the Fourier transform based model has lower estimation error for 2-ray with delay spread of $5 \mu \mathrm{s}$. This is because that the polynomial model has less model error than the Fourier transform based model when there are paths of the channel that are not at the sampling instances of the system. However, for the special case of 2-ray with delay spread of $5 \mu \mathrm{s}$, both paths are at the sampling instances of the MCM system, the Fourier transform based model does not have model error and the MMSE estimation is achieved.

Fig. 2 (a) shows the error rate of RS code for different channel estimation of TU delay profiles. Fig. 2 (b) shows the PSNR of the received $512 \times 512$ gray image "Lena" using different channel information at the transmitter and the receiver. The image is transmitted in 500 blocks. Two types of models are used for estimation, polynomial model and Fourier transform models.

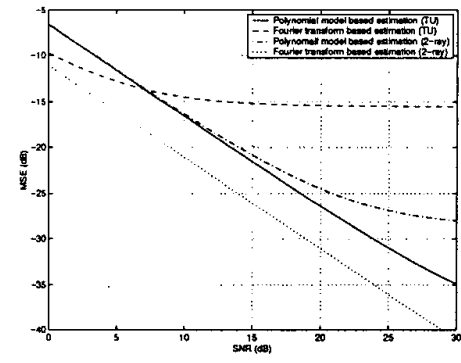

Fig. 1. Mean squared estimation error

It is usually assumed that ideal channel information is know for both transmitter and receiver. In this figure, it is shown that if the error rate information used at the transmitter is not compatible with the channel estimation method used at the receiver, the performance of the JSCM algorithm may suffer great loss. In the figure, if the receiver does not has perfect channel estimation, the PSNR results of the JSCM using error rate obtained from ideal channel information is worse than the result using error rate obtained from the compatible channel estimation. If the receiver use polynomial model based channel estimation, the estimation error is small and the PSNR results are close to the ideal results. However, the result of using compatible channel information at both transmitter and receiver is still about $1 \mathrm{~dB}$ higher than that of using ideal channel information only at the transmitter. When the channel estimation error is large, for example, using the Fourier based channel estimation at the receiver, the large discrepancy between the error rates of ideal channel information and the Fourier transform based channel estimation makes the PSNR decrease when the channel SNR increases. This means that JSCM algorithm can totally fail if the channel estimation is not considered.

Fig. 3 (a) and (b) show the WER of RS channel codes with different coding rates at SNR of $15 \mathrm{~dB}$ for both TU delay profile and 2-ray delay profile with delay spread of $5 \mu \mathrm{s}$, respectively. The results of different training densities are also shown. Fig. 3 (c) and (d) show the throughput of JSCM for a $512 \times 512$ gray image "Lena". The image is transmitted in total 500 blocks. It is shown that the polynomial model performs better than the Fourier transform model in both WER and throughput for TU delay profile while the Fourier transform model performs better for 2-ray delay profile, which is consistent with the results in Fig. 1. 


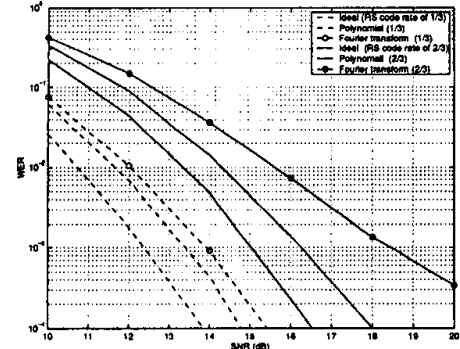

(a)

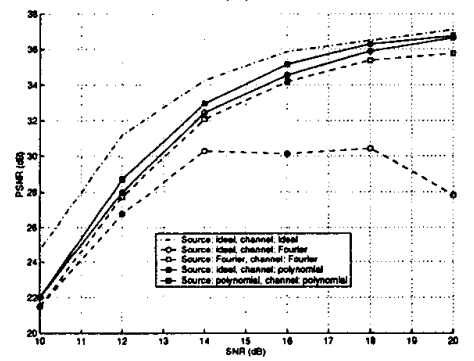

(b)

Fig. 2. (a) WER of RS code with $1 / 3$ training (TU, $f_{D}=40 \mathrm{~Hz}$ ) (b) PSNR of received Lena image

\section{CONCLUSIONS}

We proposed a model based channel estimation framework for systems with transmitter diversity in this paper. After describing the challenge of the estimation problem in such a system, we pointed out that the model based approach is not a method to improve the estimation but a necessary procedure to identify the channel. Different channel models and the identification condition are discussed based on the derived scheme.

Then we studied the effect of this channel estimation method on a robust progressive image transmission scheme using JSCM. The simulation shows that the JSCM algorithm is sensitive to the different channel estimation schemes. The channel estimation has to be applied to both the transmitter and the receiver to achieve a better performance.

\section{REFERENCES}

[1] J. A. C. Bingham, "Multicarrier Modulation for Data Transmission: An Idea Whose Time Has Come", IEEE Communications Magazine, pp5-14, May, 1990.

[2] P. A. Bello, "Characterization of Randomly Time-Variant Linear Channels", IEEE Transactions on Communications Systems, pp360-393, Dec. 1963.

[3] Ye Li, Leonard J. Cimini, Jr, and Nelson R. Sollengerger, "Robust Channel estimation for OFDM Systems with Rapid Dispersive Fading Channels", IEEE Transaction on Communications, v. 46, N. 7, pp902-915, July 1998.

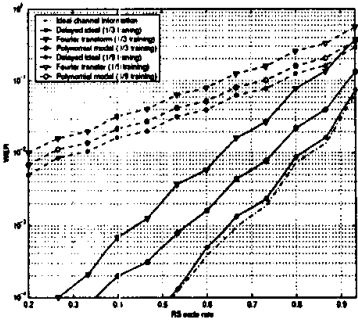

(a)

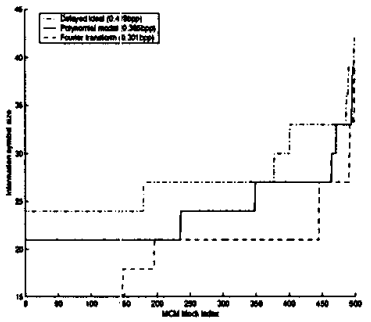

(c)

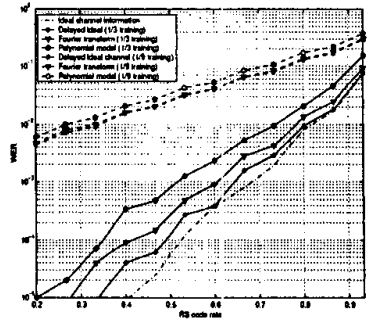

(b)

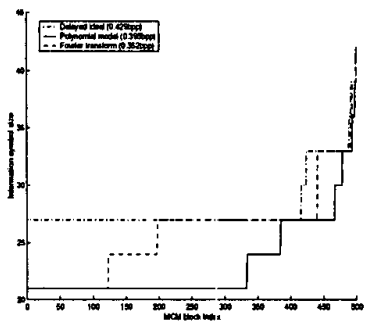

(d)
Fig. 3. WIER and throughput of JSCM using model based channel estimation ( $\mathrm{SNR}=15 \mathrm{~dB}, f_{D}=40 \mathrm{~Hz}$ ) (a) WER of RS codes (TU) (b) WER of RS codes (2-ray, $T_{d}=5 \mu \mathrm{s}$ ) (c) Throughput (TU, 1/3 training) (d) Throughput (2-ray, $T_{d}=5 \mu \mathrm{s}, 1 / 3$ training)

[4] Y. Li and N. Seshadri, "Channel Estimation for OFDM Systems with Transmitter Diversity in Mobile Wireless Channels", IEEE JSAC, v. 17, N. 3, pp461-471, March 1999.

[5] M. Luise, R. Reggiannini and G. M. Vietta, "Blind Equalization/Detection for OFDM Signals over Frequency-Selective Channels, IEEE JSAC, v. 16, N. 8, pp1568-1578, Oct. 1998.

[6] D. K. Borah and B. D. Hart, "Frequency-Selective Fading Channel Estimation with a Polynomial Time-Varying Channel Mcdel", IEEE Transactions on Communications, v. 47, N. 6, pp862-873, June 1999.

[7] S. M. Alamouti "A simple transmit diversity techniques for wireless communications", IEEE JSAC, v. 16, N. 8, Oct. 1998.

[8] V. Tarokh, H. Jafarkhani and A. R. Calderbank, "Spacetime block coding for wireless communications: performance results", IEEE JSAC, v. 17, N. 3, March 1999.

[9] A. Narula, M. D. Trott and G. W. Wornell, "Performance limits of coded diversity methods for transmitter antenna arrays", IEEE Transactions on Information Theory, v. 45, N. 7 , Nov 1999.

[10] V. Chande and N. Farvardin, "Joint Source-Channel Coding for Progressive Transmission of Embedded Source Coders", in DCC'99.

[11] J. Song and K. J. R. Liu, "Robust progressive image transmission over OFDM systems using space-time block coder EDICS: 5-WRLS", submitted to IEEE Transactions on Multimedia.

[12] J. M. Shapiro, "Embedded image coding using zerotrees of wavelet coefficients", IEEE Trans. on Signal F'rocessing, v. 41, N. 12, , pp3445-3462, Dec. 1993.

[13] A. Said and W. A. Pearlman, "A new fast and efficient image codec based on set partitioning in hierarchical irees", IEEE Trans. on CSVT, v.6, June 1996.

[14] W. C. Jakes, Microwave Mobile Communications, New York: Wiley, 1974. 